\title{
ABFM to Simplify Maintenance of Certification (MOC) for Family Physicians and Make It More Meaningful: A Family Medicine Registry
}

Robert Phillips, MD, MSPH

The American Board of Family Medicine (ABFM) launches the start of a family medicine registry with a study called the Trial of Aggregate Data Exchange for Maintenance of Certification and Raising Quality (TRADEMaRQ). This study is supported by the US Agency for Healthcare Research and Quality and is the first phase of a nearly $\$ 7$ million investment by the ABFM to make maintenance of certification (MOC) easier and more meaningful, to help physicians turn their electronic health record (EHR) data into useful information, and to support the Physician Quality Reporting System (PQRS), meaningful use, and other reporting needs. The ABFM is the first board to sponsor a registry, and this is the first clinical registry to support MOC.

\section{TRADEMaRQ: Registry Phase I}

TRADEMaRQ is the first stage of the registry and will test a way to automatically collect data for the quality improvement (QI) portion of MOC-not just for a handful of patients, but for all patients in a panel. This relieves doctors of the time-consuming task of collecting and submitting the information manually. TRADEMaRQ also will give physicians feedback about their performance as they do MOC activities to help them determine where they can make the greatest improvements, for example, by increasing rates of recommended vaccinations or cancer screenings or ensuring consistent medication use by patients with chronic conditions. The TRADEMaRQ research team will study whether performance feedback affects the self-assessment modules and practice performance modules physi-

Conflict of interest: The author is employed by the ABFM. Corresponding author: Robert Phillips, MD, MSPH, American Board of Family Medicine, 1648 McGrathiana Parkway, Suite 550, Lexington, KY 40511 (E-mail: bphillips@theabfm.org). cians choose and whether it improves quality. TRADEMaRQ as a win-win-win proposition for family physicians who already receive quality measures from their system or EHR by streamlining submission for MOC-no more entering data by hand. The ABFM and researchers will learn what might help providers quickly and effectively improve their practice, and patients will benefit from physicians who have both up-to-date medical knowledge and more time available to take care of patients.

\section{TRADEMaRQ has 3 aims:}

1. To assess whether quality measures of family physicians' entire patient panels can be shared easily with a certifying board

2. To study whether family physicians' ability to view quality measures and make comparisons with their peers will affect the types of selfassessment modules and QI efforts they choose

3. To study whether viewing quality measures and making comparisons with peers will influence the degree to which QI efforts improve future performance

The ABFM is launching TRADEMaRQ because many of its 85,000 certified family physicians already use quality measures to assess and guide QI and patient panel management in their practices. Having these whole-panel measures and then being required to enter manually quality measures for 10 of their patients for MOC made little sense.

Four health systems are participating in TRADEMaRQ: Kaiser Permanente Colorado, OCHIN, South East Texas Medical Associates, and the Group Health Cooperative. These study partners see the benefit of MOC for their family physicians and hope it will offer them a broader choice of QI options. 


\section{DAIQUERI: Registry Phase II}

The second stage of the family medicine registry is the Data Abstraction and Intelligence Quality Engine for Research and Improvement (DAIQUERI). DAIQUERI will help physicians who cannot turn their EHR data into useful information or report their measures when they need to. DAIQUERI will extract EHR data to produce patient-level quality measures and disease registries so that family physicians can improve their practice. DAIQUERI also will enable family physicians to send their measures for MOC and other reporting requirements. DAIQUERI is being developed by FIGmd (Schaumburg, IL), whose tools are currently being used to meet PQRS reporting needs for thousands of physicians. DAIQUERI will be piloted in the summer of 2015, focusing on small practices as a priority. The ABFM committed to financially supporting the first 6000 family physicians to enroll. Beyond that, we aim to keep costs far lower than those EHR vendors charge for data analytics and exchange tools and lower than the average mobile phone bill. Some other professional society-sponsored registries charge much more or they negotiate for pharma sponsorship in exchange for data. The ABFM will not engage in any data-sharing relationships that put family physician data at risk and will give diplomates control over who has access to their data and where it is sent.

\section{Which QI Tools Matter?}

Another benefit of the registry is that it will allow the ABFM to assess the benefits of various QI efforts. About half of family physicians currently do an approved MOC QI project designed by an organization other than the ABFM. We currently have no way of knowing whether those projects improve quality. The registry will eventually allow physicians to choose more reliable QI tools that make a difference.

\section{More Meaningful Primary Care Measures}

TRADEMaRQ and DAIQUERI are designed to reduce MOC burden, minimize the burden of quality measure reporting for payment, and improve ABFM's capacity to support QI, but we expect that they also will help us provide leadership in developing more meaningful primary care measures. This leadership will be important as payers shift to value-based payments. In building the registry, we recognize that there are hundreds of primary care measures but no strategy for understanding which are most valuable. Diplomates tell us they are struggling to keep up, and there is considerable measurement fatigue. We also recognize that some of the most important primary care functions have no measures. The ABFM will be working closely with Family Medicine for America's Health to understand which measures are most important and advocating for new high-value measures. The family medicine registry will strengthen the specialty's position in negotiations with measure developers and payers.

\section{An Investment in Family Medicine}

The ABFM considers the registry to be an important investment in family medicine. We recognize that PQRS, meaningful use, and other reporting requirements threaten reductions in reimbursement or public embarrassment for nonreporting. The family medicine registry will reduce these risks. The ABFM also has partnered with the American Academy of Family Physicians (AAFP) in submitting a Supporting and Aligning Network grant to the Center for Medicare and Medicaid Innovations (CMMI) to use the registry to support practice transformation networks. The ABFM is offering to triple the requested CMMI funding to align the registry and new MOC tools with practice transformation. We know that family medicine practices lack fundamental data support for a shift to value-based payments, and we see this contract as a way to move the registry and MOC into a supporting role. It also will allow us to work closely with the AAFP in developing these supports. We hope to hear from the CMMI soon about their decision.

\section{New Tools for Community and Population Health}

The AAFP's Robert Graham Center has been very successful in developing geographic data tools to help community health centers all over the United States turn their patient data into maps that demonstrate who and where they serve, where access to health care remains poor, and how social determinants of health affect their communities. ABFM leaders helped develop those tools and now want to offer them to all family physicians. The ABFM is proposing to work with the Graham Center and 
HealthLandscape, its sister center in the AAFP, to develop the Population Health Assessment Tool (PHAsT). Family physicians enrolled in the registry would have the option of using PHAsT to define their clinical service area, understand which neighborhoods are most dependent on them, look at disease and quality clusters ("hotspots"), draw on social determinant data to develop community vital signs for patients, and look at the risk of poor health across their community. Value-based payments mean greater accountability for population health, and most family physicians lack the tools or the ability to use their data to inform this role. Many family physicians also are frustrated by patients who cannot or will not take their medication or change health behaviors, often because they do not understand what is going on in their patients' lives or because they do not know who to partner with in the community to help their patients. PHAsT aims to help family physicians do population health better and improve understanding of community supports. For example, PHAsT may help you recognize that most of your patients with diabetes and hemoglobin A1c values >9.0 live in neighborhoods that are unsafe for exercise and lack healthy food sources. You could partner with a local YMCA, mall, or armory to develop an indoor walking club, diabetes peer-support group, or farmers' market-or all 3. We know from experience that family physicians in community health centers have used these tools in clever ways, and now we want all family physicians to have the same opportunity, especially when payments may soon be based on population health outcomes.

\section{An Invitation}

TRADEMaRQ, DAIQUERI, and PHAsT are a collective commitment that the ABFM is making to make MOC less burdensome, more meaningful, and a source of information that supports understanding and improvement in family medicine. This registry and its tools aim to help you do the right thing at the right time and earn more for it. The ABFM is actively seeking partners to help turn clinical data into tools that enhance primary care's impact on health. We welcome questions, concerns, advice, or offers to collaborate.

Acknowledgement: TRADEMaRQ was supported by grant number R21HS022583 from the Agency for Healthcare Research and Quality. The content is solely the responsibility of the authors and does not necessarily represent the official views of the Agency for Healthcare Research and Quality. 